\title{
FIXAÇÃO INTRAMEDULAR DAS FRATURAS DO FÊMUR NA INFÂNCIA E NA ADOLESCÊNCIA COM HASTES FLEXIVVEIS
}

\author{
INTRAMEDULLARY FIXATION OF FEMUR FRACTURES IN CHILDREN AND \\ ADOLESCENTS WITH FLEXIBLE RODS
}

César Janovsky' , Alexandre Yoiti Aoyagui', Rafael Pierami' ${ }^{1}$ Eduardo Abdalla Saad², Eiffel Tsuyoshi Dobashi², José Antonio Pinto ${ }^{3}$, Carlo Milani ${ }^{4}$

\section{RESUMO}

Objetivos: Avaliar o resultado do tratamento cirúrgico da fratura diafisária isolada do fêmur pela fixação intramedular retrógrada com hastes de Ender. Métodos: Os autores realizam um estudo retrospectivo de 31 pacientes (32 fêmures), sendo 22 (71\%) do sexo masculino e nove $(29 \%)$, do feminino. As idades variaram entre oito e 16 anos com média de 11,3 anos. Quanto ao mecanismo de trauma, observamos: 13 (42\%) foram vítimas de acidente automobilístico; cinco (16\%), de acidente motociclístico; quatro (13\%), de atropelamento; seis $(19 \%)$, de queda de altura; e três $(10 \%)$, de queda de bicicleta. Quanto ao lado acometido, ocorreram 14 (44\%) fraturas femorais direitas e 18 (56\%) esquerdas com um paciente fraturado bilateralmente. O tempo de seguimento variou de 1,6 anos a 6,3 anos com média de 2,7 anos. O período de internação foi, em média, de 6,81 dias, variando entre três e 19 dias. Após um ano, realizaram avaliação funcional (dor, movimento, claudicação) e escanometria para avaliar possíveis discrepâncias. Resultados: 28 (90,3\%) pacientes com resultado satisfatório e três $(9,6 \%)$, insatisfatório. Como complicações, observamos anisomelia em oito $(25,8 \%)$, dor em dois $(6,4 \%)$ e limitação do arco de movimento de flexão joelho em um $(3,2, \%)$ paciente. Conclusões: Os autores ainda consideram a população estudada pequena, mas os resultados demonstram ser promissores. $\mathrm{O}$ tratamento com haste intramedular de Ender foi efetivo, seguro e econômico, podendo ser indicado para as fraturas femorais isoladas estáveis.

Descritores - Fraturas do Fêmur; Fixação Intramedular de Fraturas; Procedimentos Cirúrgicos Operatórios

\section{ABSTRACT}

Objective: To evaluate the surgical treatment results using intramedullary retrograde Ender nails in isolated unilateral femur fractures. Methods: the authors performed a retrospective study of 31 patients (32 femurs), 22 (71\%) males and 9 (29\%) females with the mean age of 11,3 years (8 to 16 years old). According to the trauma mechanism we observed: 13 (42\%) car accidents, 5 (16\%) motorcycle accidents, 4 (13,\%) running over accidents, 6 (19\%) falls from height and 3(10\%) bicycle falls. The follow up varied from 1,6 to 6,3 years (average of 2,7 years). The period of hospitalization was 6,81 days varying from 3 to 19 days. After 1 year we performed clinical evaluation (pain, range of motion, gait) and scanogram do evaluate LDD. Results: We obtained 28 (90,3\%) satisfactory and 3 (9,6\%) unsatisfactory results. The following complications were recognized: LLD in 8 (25,8\%), pain 2(6,4\%), limited range of knee flexion in 1 (3,2,\%) patients. Conclusions: the authors still consider small the population studied although the higher rate of satisfactory results. The treatment with Ender nails was effective, safe, unexpensive and can be indicated to treat isolated stable femur fractures.

Keywords - Femoral Fractures; Fracture Fixation, Intramedullary; Surgical Procedures, Operative

1 - Residente do $3^{\circ}$ ano do Departamento de Ortopedia e Traumatologia da EPM/Unifesp - São Paulo, SP, Brasil.

2 - Doutor da Disciplina de Ortopedia Pediátrica do Departamento de Ortopedia e Traumatologia da EPM/Unifesp - São Paulo, SP, Brasil.

3 - Professor Adjunto; Chefe de Clínica da Disciplina de Ortopedia Pediátrica do Departamento de Ortopedia e Traumatologia da EPM/Unifesp - São Paulo, SP, Brasil.

4 - Professor do Departamento de Ortopedia e Traumatologia da EPM/Unifesp - São Paulo, SP, Brasil.

Trabalho realizado na Disciplina de Ortopedia Pediátrica do Departamento de Ortopedia e Traumatologia da EPM/Unifesp.

Correspondência: Rua Borges Lagoa, 783, 5ªndar, Vila Clementino - 04038-032 - São Paulo, SP. E-mail: janovsky@unifesp.br

Trabalho recebido para publicação: 24/08/2011, aceito para publicação: 31/08/2011.

Os autores declaram inexistência de conflito de interesses na realização deste trabalho / The authors declare that there was no conflict of interest in conducting this work 


\section{INTRODUÇÃO}

As fraturas do fêmur antes da maturidade esquelética correspondem a, aproximadamente, $1,6 \%$ de todas as lesões ósseas dessa população.

Sua distribuição é bimodal, observando-se um pico aos dois anos (provocado por traumas de baixa energia devido às características anatômicas e biomecânicas do esqueleto nessa idade) e outro na adolescência (relacionado a acidentes de alta energia, como os automobilísticos, e frequentemente há associação com lesões de outros segmentos corporais, como os torácicos, cranianos e abdominais).

A terapêutica dessas fraturas, especialmente as da diáfise femoral, gera polêmica entre os diversos autores ${ }^{(1,2)}$. Os que defendem o tratamento incruento baseiam-se na observância dos altos índices de resultados satisfatórios obtidos. Outros, advogam que a definição do tratamento deve considerar a idade do paciente, as características da fratura, os traumas associados, as possíveis doenças preexistentes e, por fim, os fatores socioeconômicos ${ }^{(2,3)}$.

O tratamento usual que prevalece para as crianças com idade até 10 anos é o não operatório ${ }^{(4,5)}$, utilizando-se a tração cutânea ou a esquelética, o gesso pelvipodálico imediato ou tardio e as órteses. Entretanto, a partir de 1980, com o aperfeiçoamento das técnicas de osteossíntese e a introdução de novos conceitos biomecânicos, diversos autores têm relatado bons resultados de acordo com suas experiências utilizando-se do tratamento cirúrgico ${ }^{(3,4,6,7)}$. Outro aspecto observado é a progressiva diminuição das faixas etárias em que o tratamento operatório têm sido aplicado.

Este trabalho foi realizado com o objetivo de demonstrar os resultados do tratamento cirúrgico pela fixação retrógrada intramedular com hastes flexíveis de Ender.

\section{MATERIAL E MÉTODOS}

O projeto deste estudo foi analisado e aprovado pelo Comitê de Ética Médica de nossa instituição, registrado sob o número 0481/02.

Foram estudadas, entre janeiro de 1998 e outubro de 2002, 32 fraturas diafisárias do fêmur de 31 pacientes submetidos ao tratamento cirúrgico aplicando-se as hastes intramedulares de Ender.

Dos 31 pacientes estudados, 22 (71\%) eram do sexo masculino e nove (29\%), do feminino. As idades variaram entre oito e 16 anos com média de 11,3 anos. Em relação ao mecanismo de trauma, 13 (42\%) foram vítimas de acidente automobilístico, cinco (16\%) de acidente motociclístico, quatro (13\%) de atropelamento, seis $(19 \%)$ de queda de altura e três $(10 \%)$ de queda de bicicleta. Quanto ao lado acometido, ocorreram 14 (44\%) fraturas femorais direitas e 18 (56\%) esquerdas com um paciente fraturado bilateralmente.

O período de internação de nossos pacientes foi em media 6,81 dias, variando entre três e 19 dias (Quadro 1).

Foram realizados exames radiográficos convencionais nas incidências anteroposterior e perfil para o diagnóstico e análise das fraturas. $\mathrm{Na}$ admissão, todas as fraturas foram estabilizadas por meio da tração cutânea, mantendo o membro inferior acometido sobre uma férula. A tração esquelética foi instalada quando o procedimento cirúrgico não pode ser realizado nas primeiras 48 horas, utilizando um contrapeso equivalente a $10 \%$ do peso corporal do paciente.

\section{MÉTODO CIRÚRGICO}

$\mathrm{Na}$ avaliação pré-operatória escolhemos o tamanho da haste a partir da análise da radiografia inicial do fêmur fraturado, ou, quando necessário, pela avaliação radiográfica do fêmur contralateral.

No planejamento operatório o comprimento da haste a ser introduzida lateralmente foi determinado pela medida da distância entre a base do trocanter maior e a fise lateral distal do fêmur.

A medida da haste medial foi definida pela mensuração da distância entre a fise femoral proximal e a fise medial e distal. O diâmetro foi determinado pela medida a partir da largura do canal medular ao nível do istmo femoral. Hastes de 3,5 e 4,5mm de diâmetro foram utilizadas.

Com o paciente devidamente anestesiado, posicionamos o paciente em decúbito dorsal horizontal em uma mesa cirúrgica radiotransparente ou mesa ortopédica. $\mathrm{O}$ preparo da pele do membro inferior acometido consistiu da aplicação de solução de PVP-I (Polivinil Pirrolidona Iodo) degermante antecedida pela tricotomia, quando necessária. Realizamos a antissepsia com PVP-I alcoólico e, em seguida, colocamos os campos cirúrgicos estéreis e a malha tubular. Realizamos uma incisão de aproximadamente $2 \mathrm{~cm}$ ao nível do côndilo femoral lateral.

Aprofundamos o bisturi cranialmente, seccionando o tecido celular subcutâneo, a fascia lata, o músculo vasto lateral e o periósteo. Sob visão radioscópica, perfuramos a cortical lateral do fêmur com sovela proximalmente a, aproximadamente, $2 \mathrm{~cm}$ da placa de crescimento. 
Quadro 1 - Distribuição dos pacientes segundo o número de ordem, sexo, idade em anos, lado acometido, mecanismo de trauma, traumas associados, tempo de seguimento e tempo de hospitalização.

\begin{tabular}{|c|c|c|c|c|c|c|c|}
\hline Ordem & Sexo & Idade & Lado & Mecanismo de trauma & Traumas associados & \begin{tabular}{|c|}
$\begin{array}{c}\text { Tempo de seguimento } \\
\text { (anos) }\end{array}$ \\
\end{tabular} & $\begin{array}{l}\text { Hospitalização } \\
\text { (dias) }\end{array}$ \\
\hline 1 & $\mathrm{~F}$ & 9,3 & $\mathrm{D}$ & Atropelamento & TCE + fratura da mandíbula & 6,2 & 12 \\
\hline 2 & M & 10,4 & $\mathrm{D}$ & Queda de bicicleta & - & 2,5 & 4 \\
\hline 3 & $\mathrm{~F}$ & 10 & $\mathrm{D}$ & Automobilístico & - & 1,9 & 5 \\
\hline 4 & $M$ & 13,6 & $\mathrm{D}$ & Motocicleta & - & 2,5 & 8 \\
\hline 5 & $\mathrm{M}$ & 10,2 & $\mathrm{D}$ & Queda de bicicleta & - & 4,5 & 3 \\
\hline 6 & $M$ & 15 & $E$ & Queda de altura & - & 2,7 & 9 \\
\hline 7 & $\mathrm{M}$ & 12,3 & $E$ & Motocicleta & - & 3,1 & 7 \\
\hline 8 & $\mathrm{M}$ & 10,2 & $\mathrm{D}$ & Automobilístico & - & 2,2 & 10 \\
\hline 9 & $M$ & 11,6 & $B$ & Atropelamento & TCE & 6,2 & 6 \\
\hline 10 & M & 8,2 & $E$ & Automobilístico & $\begin{array}{l}\text { TCE + ruptura do baço, } \\
\text { fratura de clavícula }\end{array}$ & 2,1 & 13 \\
\hline 11 & $\mathrm{~F}$ & 10,5 & $E$ & Queda de altura & Contusões múltiplas & 3,5 & 5 \\
\hline 12 & $M$ & 11,7 & $E$ & Queda de altura & TCE & 01/jun & 6 \\
\hline 13 & M & 11,9 & $E$ & Queda de altura & $\begin{array}{c}\text { TCE+ FCC infectado na } \\
\text { axila D }\end{array}$ & 2,5 & 19 \\
\hline 14 & $\mathrm{~F}$ & 10 & $E$ & Automobilístico & - & 2,3 & 4 \\
\hline 15 & $M$ & 11,6 & $\mathrm{D}$ & Atropelamento & TCE & 2,5 & 6 \\
\hline 16 & $\mathrm{~F}$ & 12 & D & Automobilístico & - & 2,9 & 8 \\
\hline 17 & $\mathrm{~F}$ & 10 & $E$ & Automobilístico & - & 2,1 & 5 \\
\hline 18 & $M$ & 10,1 & $\mathrm{D}$ & Automobilístico & - & 2,3 & 7 \\
\hline 19 & $\mathrm{~F}$ & 10 & $E$ & Automobilístico & Fratura de quadril & 2,7 & 5 \\
\hline 20 & $\mathrm{M}$ & 12 & $\mathrm{D}$ & Automobilístico & - & 1,6 & 9 \\
\hline 21 & $M$ & 11,7 & $E$ & Automobilístico & - & 2,3 & 3 \\
\hline 22 & M & 12 & $\mathrm{D}$ & Automobilístico & $\begin{array}{l}\text { Fratura do úmero } E \text {, lesão } \\
\text { face e olhos }\end{array}$ & 2,2 & 9 \\
\hline 23 & M & 12,9 & $\mathrm{D}$ & Motocicleta & TCE + contusões múltiplas & 2,1 & 4 \\
\hline 24 & $\mathrm{~F}$ & 12,5 & $\mathrm{E}$ & Motocicleta & Fratura da tíbia & 1,8 & 4 \\
\hline 25 & $\mathrm{~F}$ & 10,6 & $E$ & Queda de altura & Contusões múltiplas & 05/jan & 3 \\
\hline 26 & M & 13 & $E$ & Motocicleta & $\begin{array}{l}\text { Fraturas do pé } \mathrm{E} \text {, ulna } \mathrm{E}, \\
\text { radio } \mathrm{D}\end{array}$ & 4,5 & 3 \\
\hline 27 & M & 9,5 & $\mathrm{D}$ & Atropelamento & & 2 & 10 \\
\hline 28 & M & 11,2 & $E$ & Automobilístico & & 2,3 & 8 \\
\hline 29 & M & 11,5 & $E$ & Automobilístico & Contusões múltiplas & 6,8 & 6 \\
\hline 30 & M & 9,3 & $\mathrm{E}$ & Queda de bicicleta & TCE & 5,8 & 3 \\
\hline 31 & M & 15,9 & $E$ & Queda de altura & & 5,2 & 7 \\
\hline
\end{tabular}

$\mathrm{M}=$ masculino; $\mathrm{F}$ = feminino; $\mathrm{D}$ = direito; $\mathrm{E}$ = esquerdo; $\mathrm{B}=$ bilateral; $\mathrm{TCE}$ = traumatismo craniencefálico; $\mathrm{FCC}$ = ferimento cortocontuso

A haste de Ender era então introduzida pelo canal medular sob visão radioscópica atravessando o foco da fratura. A extremidade proximal da síntese foi direcionada para o trocanter maior, preservando-se, desta forma, a fise de crescimento desta estrutura óssea. Uma segunda incisão cirúrgica foi feita na face medial do joelho e foram executados procedimentos semelhantes aos que foram realizados na face lateral. Direcionamos a segunda haste em direção ao colo do fêmur, mantendo uma distância de, aproximadamente, $2 \mathrm{~cm}$ da fise de crescimento. Realizamos uma radiografia de controle após a fixação do fêmur, hemostasia cuidadosa, fechamento dos tecidos por planos e, por fim, aplicamos curativos oclusivos nas feridas cirúrgicas. Não utilizamos drenos de aspiração no período pós-operatório.

O membro inferior foi mantido apoiado em uma férula; a mobilização precoce do joelho e do quadril estimulada conforme a tolerância do paciente; e a deambulação com o auxílio de muletas foi permitida após as primeiras 24 horas do período pós-operatório.

A avaliação clínica e radiográfica foi realizada semanalmente, até observarmos a consolidação das fraturas, permitindo, assim, deambulação com carga total sobre o membro operado. O início da deambulação sem muletas 
ocorreu entre quatro e seis semanas, e a remoção das hastes entre seis e 12 meses.

Após um período mínimo de um ano, cada paciente foi submetido à escanometria, para a mensuração dos membros inferiores. Os resultados funcionais nesta mesma ocasião levaram em consideração a presença de dor, o arco de movimento articular do quadril e joelho, o comprimento entre os membros inferiores, os desvios angulares (varo ou valgo da diáfise femoral) e/ou rotacionais (mensuração comparativa das rotações interna e externa máximas dos quadris) e a avaliação visual da função da marcha.

A avaliação funcional dos pacientes estudados considerou as variáveis acima analisadas e classificamos os resultados em satisfatório e insatisfatório. Caracterizamos o resultado como insatisfatório quando fossem evidenciadas as seguintes situações, reconhecidas isoladamente ou combinadas com outras alterações: presença de dor, presença de limitação do arco de movimento, deformidades angulares e/ou rotacionais que determinem tratamentos cirúrgicos complementares, anisomelia superior a $2 \mathrm{~cm}$, claudicação.

\section{MÉTODO ESTATÍSTICO}

As variáveis qualitativas foram representadas por frequência absoluta (n) e relativa (\%) e as quantitativas, por média e desvio padrão. Para avaliarmos a possível diferença quanto ao tempo de internação entre os grupos sem trauma associado e com trauma associado, usamos o teste $t$ de Student para duas amostras independentes.

Para verificarmos se o tempo de internação assim obtido foi diferente do tempo de internação obtido por outros autores, usamos o teste $t$ médio amostral em relação à média populacional. Em todos os casos, o nível de significância para rejeição da hipótese de nulidade foi fixado em 0,05 (5\%). Quando a estatística calculada apresentou significância, usamos um asterisco $\left(^{*}\right)$ para caracterizá-la.

\section{RESULTADOS}

Como complicações, observamos anisomelia em oito $(25,8 \%)$, dor em dois $(6,4 \%)$ e limitação do arco de movimento de flexão joelho em um $(3,2, \%)$ paciente.

A distribuição dos pacientes de acordo com o número de ordem, discrepância de comprimento entre os membros inferiores, complicações associadas, desvio angular, desvio rotacional, análise visual da marcha e o resultado final é demonstrada no Quadro 2. A anisomelia determinada pelo hipercrescimento femoral do lado operado foi reconhecida em oito $(25,8 \%)$ pacientes. A dor do membro inferior foi reconhecida em dois $(6,4 \%)$ pacientes, e a limitação do arco de movimento de flexão joelho (menor que 10 graus comparado com o lado contralateral) foi observada em um $(3,2, \%)$ indivíduo. Não foi reconhecida clinicamente alterações angulares, rotacionais ou da análise visual da marcha.

Para a análise funcional dos resultados, obtivemos $28(90,3 \%)$ pacientes com resultado satisfatório e três $(9,6 \%)$, insatisfatório.

Na Tabela 1, comparamos os pacientes com e sem traumas associados, segundo o tempo de internação em dias.

Na Tabela 2, apresentamos a distribuição dos pacientes sem discrepância entre os membros inferiores, com até $0,5 \mathrm{~cm}$ e entre 0,6 e $1,0 \mathrm{~cm}$ em relação à idade.

$\mathrm{Na}$ Tabela 3, apresentamos os valores do custo, em média utilizando como referência a tabela SUS e $\mathrm{AMB} / 92(\mathrm{CH}=0,30)$ e os valores estimados considerando o sistema privado (convênio e particular). Foram considerados: diárias do paciente e acompanhante, serviços hospitalares, serviços de enfermagem e médico (com exceção do particular), alimentação do paciente e acompanhante, taxa de sala cirúrgica, monitor cardíaco, oxigênio, medicamentos, material ortopédico, haste intramedular de Ender, radiografia e radioscopia.

Tabela 1 - Pacientes segundo o tempo de internação, em dias, sem e com trauma associado e resultado da estatística.

\begin{tabular}{|c|c|c|c|c|c|}
\hline \multicolumn{3}{|c|}{ Sem trauma } & \multicolumn{3}{|c|}{ Com trauma } \\
\hline № & Iniciais & $\begin{array}{c}\text { Tempo } \\
\text { (dias) }\end{array}$ & $\mathrm{N}^{\circ}$ & Iniciais & $\begin{array}{c}\text { Tempo } \\
\text { (dias) }\end{array}$ \\
\hline 2 & Adnp & 4 & 1 & Acmal & 12 \\
\hline 3 & Asm & 5 & 9 & Ejjrr & 6 \\
\hline 4 & Bbn & 8 & 10 & Ejk & 13 \\
\hline 5 & Ccp & 3 & 11 & Erm & 5 \\
\hline 6 & Cor & 9 & 12 & Esa & 6 \\
\hline 7 & Dms & 7 & 13 & Ets & 19 \\
\hline 8 & Dsam & 10 & 15 & Flps & 6 \\
\hline 14 & Fan & 4 & 19 & Maf & 5 \\
\hline 16 & Gdr & 8 & 22 & Poq & 9 \\
\hline 17 & Jls & 5 & 23 & Rfvn & 4 \\
\hline 18 & Jss & 7 & 24 & Rja & 4 \\
\hline 20 & Mms & 9 & 25 & Rmf & 3 \\
\hline 21 & Plut & 3 & 26 & Rspm & 3 \\
\hline 27 & Sad & 10 & 29 & Sdrb & 6 \\
\hline 28 & Scr & 8 & 30 & Vdr & 3 \\
\hline 31 & Wro & 7 & & & \\
\hline \multicolumn{2}{|c|}{$N=16$} & lédia = 6,9 & \multicolumn{2}{|c|}{$N=15$} & $i a=6,7$ \\
\hline \multicolumn{2}{|c|}{ Grupo } & \multicolumn{2}{|l|}{$\mathbf{N}$} & $\begin{array}{c}\text { Média de } \\
\text { internação }\end{array}$ & $\begin{array}{l}\text { Desvio } \\
\text { padrão }\end{array}$ \\
\hline \multicolumn{2}{|c|}{ Com trauma } & \multicolumn{2}{|l|}{15} & 6,9 & 4,52 \\
\hline \multicolumn{2}{|c|}{ Sem trauma } & 16 & & 6,7 & 2,38 \\
\hline
\end{tabular}


Quadro 2 - Distribuição dos pacientes segundo o número de ordem, discrepância de comprimento entre os membros inferiores, complicações associadas, desvio angular, desvio rotacional, análise visual da marcha e o resultado final.

\begin{tabular}{|c|c|c|c|c|c|c|}
\hline $\begin{array}{l}\text { Número de } \\
\text { ordem }\end{array}$ & Anisomelia & Complicações & Desvio angular & Desvio rotacional & Marcha & Resultado funcional \\
\hline 1 & Não & Não & Não & Não & Sem claudicação & Satisfatório \\
\hline 2 & $+0,5 \mathrm{~cm}$ & Não & Não & Não & Sem claudicação & Satisfatório \\
\hline 3 & $+1,0 \mathrm{~cm}$ & $\begin{array}{l}\text { Limitação da } \\
\text { flexão do joelho }\end{array}$ & Não & Não & Sem claudicação & Insatisfatório \\
\hline 4 & Não & Não & Não & Não & Sem claudicação & Satisfatório \\
\hline 5 & $+0,5 \mathrm{~cm}$ & Não & Não & Não & Sem claudicação & Satisfatório \\
\hline 6 & Não & Não & Não & Não & Sem claudicação & Satisfatório \\
\hline 7 & Não & Não & Não & Não & Sem claudicação & Satisfatório \\
\hline 8 & Não & Não & Não & Não & Sem claudicação & Satisfatório \\
\hline 9 & Não & Não & Não & Não & Sem claudicação & Satisfatório \\
\hline 10 & Não & Não & Não & Não & Sem claudicação & Satisfatório \\
\hline 11 & Não & Não & Não & Não & Sem claudicação & Satisfatório \\
\hline 12 & Não & Não & Não & Não & Sem claudicação & Satisfatório \\
\hline 13 & $+1,0 \mathrm{~cm}$ & Não & Não & Não & Sem claudicação & Satisfatório \\
\hline 14 & $=1,0 \mathrm{~cm}$ & Não & Não & Não & Sem claudicação & Satisfatório \\
\hline 15 & Não & Não & Não & Não & Sem claudicação & Satisfatório \\
\hline 16 & $+0,5 \mathrm{~cm}$ & Dor no joelho & Não & Não & Sem claudicação & Insatisfatório \\
\hline 17 & Não & Não & Não & Não & Sem claudicação & Satisfatório \\
\hline 18 & Não & Não & Não & Não & Sem claudicação & Satisfatório \\
\hline 19 & $+0,5 \mathrm{~cm}$ & Não & Não & Não & Sem claudicação & Satisfatório \\
\hline 20 & $+1,0 \mathrm{~cm}$ & Dor no joelho & Não & Não & Sem claudicação & Insatisfatório \\
\hline 21 & Não & Não & Não & Não & Sem claudicação & Satisfatório \\
\hline 22 & Não & Não & Não & Não & Sem claudicação & Satisfatório \\
\hline 23 & Não & Não & Não & Não & Sem claudicação & Satisfatório \\
\hline 24 & Não & Não & Não & Não & Sem claudicação & Satisfatório \\
\hline 25 & Não & Não & Não & Não & Sem claudicação & Satisfatório \\
\hline 26 & Não & Não & Não & Não & Sem claudicação & Satisfatório \\
\hline 27 & Não & Não & Não & Não & Sem claudicação & Satisfatório \\
\hline 28 & Não & Não & Não & Não & Sem claudicação & Satisfatório \\
\hline 29 & Não & Não & Não & Não & Sem claudicação & Satisfatório \\
\hline 30 & Não & Não & Não & Não & Sem claudicação & Satisfatório \\
\hline 31 & Não & Não & Não & Não & Sem claudicação & Satisfatório \\
\hline
\end{tabular}

Tabela 2 - Pacientes submetidos ao tratamento com haste flexível de Ender segundo a idade, em anos, e o grau de discrepância dos membros inferiores.

\begin{tabular}{|c|c|c|c|c|c|c|c|c|}
\hline \multirow{3}{*}{ Idade } & \multicolumn{8}{|c|}{ Discrepância Mmii } \\
\hline & \multicolumn{2}{|c|}{ Sem } & \multicolumn{2}{|c|}{$0,5 \mathrm{~cm}$} & \multicolumn{2}{|c|}{$1,0 \mathrm{~cm}$} & \multicolumn{2}{|c|}{ Total } \\
\hline & \multicolumn{2}{|c|}{$\mathbf{N} \%$} & \multicolumn{2}{|c|}{$\mathrm{N} \%$} & \multicolumn{2}{|c|}{$\mathbf{N} \%$} & \multicolumn{2}{|r|}{$\mathbf{N} \%$} \\
\hline 8 & 1 & 100,0 & & & & & 1 & 100,0 \\
\hline 9 & 3 & 100,0 & & & & & 3 & 100,0 \\
\hline 10 & 5 & 50,0 & 3 & 30,0 & 2 & 20,0 & 10 & 100,0 \\
\hline 11 & 6 & 85,7 & & & 1 & 14,3 & 7 & 100,0 \\
\hline 12 & 4 & 66,7 & 1 & 16,7 & 1 & 16,7 & 6 & 100,0 \\
\hline 13 & 2 & 100,0 & & & & & 2 & 100,0 \\
\hline 15 & 2 & 100,0 & & & & & 2 & 100,0 \\
\hline Total & 23 & 74,2 & 4 & 12,9 & 4 & 12,9 & 31 & 100,0 \\
\hline
\end{tabular}

$\mathrm{N}=$ número; Mmii = membros inferiores
Tabela 3 - Relação estimada dos custos médico-hospitalares (em Reais) com o tratamento cirúrgico (fixação com haste flexível de Ender) e não cirúrgico (tração esquelética seguida de gesso) em hospital público, conveniado e privado.

\begin{tabular}{c|c|c}
\hline & Tratamento cirúrgico & $\begin{array}{c}\text { Tração esquelética } \\
\text { e gesso }\end{array}$ \\
\hline Hospital público & 514,84 & 722,49 \\
\hline Convênio enfermaria & $2.208,34$ & $4.143,37$ \\
\hline Convênio apartamento & $3.500,28$ & $7.929,25$ \\
\hline Particular & $4.047,82$ & $10.592,75$ \\
\hline
\end{tabular}




\section{DISCUSSÃO}

São múltiplos os fatores que influenciam o ortopedista na escolha do método mais apropriado de tratamento, como: a idade, o tipo de fratura, as possíveis lesões associadas, as condições socioeconômicas e psíquicas do paciente. A experiência do cirurgião e a correta seleção dos métodos e dos pacientes são de relevante importância.

Entre as indicações clássicas para a aplicação do tratamento cirúrgico na fratura diafisária do fêmur, estão: a associação das mesmas com o traumatismo craniencefálico, a presença de convulsão ou espasticidade, as fraturas expostas, a associação com o joelho flutuante e a detecção de lesões vasculonervosas ipsilaterais ${ }^{(2,5)}$. Salientamos que a falha do tratamento incruento também deve ser considerada para a mudança da modalidade terapêutica, concordando com o relato de outros autores da literatura ortopédica ${ }^{(8)}$.

Apesar do tratamento conservador ser considerado o método de eleição para as fraturas diafisárias do fêmur em crianças ${ }^{(4)}$, quando nos deparamos com um paciente politraumatizado, a indicação de cirurgia é unânime, pois, permite-se, com isso, uma manipulação apropriada para seus cuidados. O nível socioeconômico do paciente, a preferência familiar por determinada modalidade terapêutica e o alto custo ao final do tratamento são fatores que, em nosso meio, também devem ser considerados ${ }^{(6)}$.

As inúmeras complicações e os insucessos observados quando aplicado o tratamento conservador, principalmente nas crianças mais velhas ou obesas, fizeram com que diversos autores começassem a preferir as estabilizações cirúrgicas desta afecção. Entre elas, o encurtamento femoral precipitado pela ação das forças musculares e pela diminuição do fenômeno do hipercrescimento induzida pela puberdade ${ }^{(9)}$. Não observamos discrepâncias superiores a $1 \mathrm{~cm}$ após o acompanhamento mínimo de um ano.

A aplicação de um aparelho gessado antecedida pela tração quando associada com o traumatismo craniano demonstra altos índices de consolidação viciosa, chegando a $33,3 \%$ em alguns estudos ${ }^{(10)}$. Em outro trabalho, foi observado que, de 29 fraturas tratadas incruentamente com a utilização da tração esquelética aplicada na tuberosidade anterior da tíbia, 12 pacientes obtiveram resultados funcionais e radiográficos insatisfatórios por apresentarem desvios e encurtamentos com mais de $2,0 \mathrm{~cm}^{(11)}$.

As vantagens do tratamento cirúrgico nas crianças acima dos 10 anos de idade incluem: a facilidade e a precocidade na mobilização do paciente, a melhora da capacidade respiratória, a prevenção contra escaras, a minimização dos riscos de infecção pulmonar, a estabilização apropriada, a diminuição do período de hospitalização, a diminuição dos custos do tratamento ${ }^{(12)}$ e a possibilidade do retorno precoce às atividades escolares. Ressaltamos ainda a facilidade na higiene do paciente; a facilidade na remoção e mobilização, principalmente nas crianças mais velhas; a melhora da qualidade de vida e do convívio social da criança e de seus responsáveis; e o retorno às atividades escolares.

A utilização de placas de compressão dinâmica ou de neutralização têm demonstrado bons resultados com a aplicação deste método, e a técnica para sua aplicação é de domínio de todos ${ }^{(3,12)}$. Entretanto, altas taxas de infecção, associação de um grande número de lesões e queda da resistência imunológica predispõem os pacientes internados especialmente por períodos prolongados às complicações $^{(13,14)}$. Além das anisomelias, podemos observar a soltura da placa, a fratura por estresse após a remoção da síntese, bem como a necessidade de remoção da placa ${ }^{(13)}$.

Acreditamos que a utilização da via retrógrada convencional ou o duplo acesso, por nós preconizado para a colocação das hastes de Ender, pode impedir quaisquer lesões osteocartilaginosas do complexo proximal do fêmur, que são potencialmente devastadoras.

O método de introdução das hastes flexíveis de Ender, por nós utilizado, permitiu a identificação pela radioscopia das cartilagens de crescimento com segurança e consequentemente evitou possíveis alterações do crescimento.

Com a aplicação de duas hastes de Ender, medial e lateral, ressaltamos as vantagens biomecânicas deste tipo de estabilização, pois, com esta configuração, obtivemos grande estabilidade em virtude do efeito de "mola", semelhante àquele observado pela aplicação do fixador externo de Ulson para as fraturas do rádio ${ }^{(15)}$. Pela disposição das hastes, evitamos a translação entre os fragmentos, pois sabemos que a conformação de maior estabilidade é conferida quando aplicamos fios cruzados entre si. Esta maior estabilidade permitiu-nos evitar a aplicação de medidas adicionais para evitar possíveis desvios após a cirurgia. Sugerimos que a utilização de duas hastes deva ser feita somente nas fraturas com traço único ou em asa de borboleta. Não recomendamos este método nas fraturas com grande cominuição, pois seria muitíssimo difícil controlar o comprimento do fêmur.

Consideramos imprescindível o uso do aparelho de radioscopia, sem o qual seria difícil identificar com segurança, precisão e rapidez as fises, proximal e distal, possibilitando sua preservação. Consequentemente, houve uma redução do tempo cirúrgico, diminuindo-se os riscos operatórios (menor tempo de anestesia e de garrote, menor sangramento, diminuição dos riscos de infecção etc). Com isso, foi possível realizar o procedimento cirúrgico por um acesso mínimo. Esteticamente, 
nossos resultados foram bastante satisfatórios, pois nossos pacientes ficaram com uma pequena cicatriz medial e outra lateral. Em comparação com os outros métodos, ressaltamos a presença de diversas retrações cicatriciais originadas pela introdução dos pinos do fixador externo e da cicatriz retilínea longa proveniente da incisão lateral da coxa para a colocação da placa de compressão.

Estudos ainda promovem o uso de hastes flexíveis nas fraturas diafisária do fêmur como um método seguro, com uma curva de aprendizado curta e que ainda permite mobilização precoce ${ }^{(16)}$.

Os fixadores externos têm mostrado eficiência para o tratamento, tanto das fraturas múltiplas como da fratura femoral isolada ${ }^{(3)}$. Foi estabelecida arbitrariamente a idade limite de seis anos de idade para sua utilização, observando que, em crianças mais jovens, o índice de complicações é maior ${ }^{(7,17)}$. O movimento articular do quadril e do joelho podem ficar limitados em virtude das perfurações dos tecidos moles e subsequente fibrose muscular. Alguns autores referem que esta limitação articular é transitória e a recuperação ocorreria após quatro semanas da retirada do fixador. Outra complicação frequente é a infecção no trajeto dos pinos, variando de $0 \%$ a $45 \%{ }^{(3)}$. Refratura pode ocorrer em virtude de um osso regenerado pouco resistente, da consolidação viciosa ou da pseudartrose. Não tivemos problemas com a consolidação das fraturas no nosso estudo.

Quanto ao arco de movimento articular, observamos baixo índice de limitações funcionais em nossos pacientes, pois, acreditando na maior estabilidade conferida pelo método aplicado, submetemos os pacientes à fisioterapia com movimentos passivos e ativos desde o primeiro pós-operatório.

Após a análise clínica e radiográfica, verificamos resultados satisfatórios em $29(90,3 \%)$ pacientes quando utilizamos nossos critérios de avaliação. Considerando a população abordada, em virtude da grande capacidade

\section{REFERÊNCIAS}

1. Blasier RD, Aronson J, Tursky EA. External fixation of pediatric fêmur fractures. J Pediatr Orthop. 1997;17(3):342-6.

2. Herndon WA, Mahnken RF, Yngve DA, Sullivan JA. Management of femoral shaft fractures in the adolescent. J Pediatr Orthop. 1989;9(1):29-32.

3. Davis TJ, Topping RE, Blanco JS. External fixation of pediatric femoral fractures. Clin Orthop Relat Res. 1995;(318):191-8.

4. Clement DA, Colton CL. Overgrowth of the femur after fracture in childhood. $\mathrm{Na}$ increased effect in boys. J Bone Joint Surg Br. 1986;68(4):534-6.

5. Karaoğlu S, Baktir A, Tuncel M, Karakaş ES, Sakir TM. Closed Ender nailing of adolescent femoral shaft fractures. Injury. 1994;25(8):501-6.

6. Aronson J, Tursky EA. External fixation of femur fractures in children. J Pediatr Orthop. 1992;12(2):157-63.

7. Canale ST, Tolo VT. Fractures of the femur in children. Instr Course Lect.1995;44:255-73.

8. Mann DC, Weddington J, Davenport K. Closed Ender nailing of femoral shaft fractures in adolescents. J Pediatr Orthop. 1986;6(6):651-5.

9. Heinrich SD, Drvaric D, Darr K, MacEwen GD. Stabilization of pediatric diaphyseal femur fractures with flexible intramedullary nails (a technique paper). $\mathrm{J}$ Orthop Trauma. 1992;6(4):452-9. de remodelação e adaptação funcional destes indivíduos, qualquer parâmetro alterado que requeresse tratamento complementar foi categorizado como insatisfatório, em que somente os resultados muito próximos à plena normalidade pudessem ser aceitos.

Considerando o fator econômico verificamos que, após a simulação dos gastos, independente do sistema de atendimento o método cirúrgico se mostra economicamente menos dispendioso, logo este fato não pode ser menosprezado em nosso país. A associação com lesões do aparelho locomotor e de outros sistemas não determinou maior período de permanência hospitalar em nosso estudo, pois estas acabam sendo tratadas simultaneamente.

Muitos autores atualmente têm se utilizado das hastes intramedulares flexíveis de titânio, advogando a favor de que este material teria propriedades físicas de elasticidade que seriam benéficas para o osso envolvido. Infelizmente, este material apresenta custo elevado quando comparado com as hastes de aço. Estas propriedades elásticas são muito pouco aproveitadas, visto que a consolidação e o tempo de permanência das hastes no paciente pediátrico são breves.

\section{CONCLUSÕES}

O tratamento cirúrgico das fraturas femorais pela fixação intramedular retrógrada com hastes de Ender apresentou 29 (90,3\%) pacientes com resultados satisfatórios e três $(9,7 \%)$ insatisfatórios.

Como complicações observamos anisomelia em oito $(25,8 \%)$, dor em dois $(6,4 \%)$ e limitação do arco de movimento de flexão joelho em um $(3,2, \%)$ paciente.

Lesões associadas de outros sistemas e aparelhos não determinou período maior de internação.

O tratamento com haste intramedular de Ender foi efetivo, seguro e econômico, podendo ser indicado para as fraturas femorais isoladas estáveis.

10. Gregory P, Sullivan JA, Herndon WA. Adolescent femoral shaft fractures: rigid versus flexible nails. Orthopedics. 1995;18(7):645-9.

11. Galpin RD, Willis RB, Sabano N. Intramedullary nailing of pediatric femoral fractures. J Pediatr Orthop. 1994;14(2):184-9.

12. Kregor PJ, Song KM, Routt ML Jr, Sangeorzan BJ, Liddell RM, Hansen ST Jr. Plate fixation of femoral shaft fractures in multiply injured children. J Boné Joint Surg Am. 1993;75(12):1774-80.

13. Ward WT, Levy J, Kaye A. Compression plating for child and adolescent femur fractures. J Pediatr Orthop. 1992;12(5):626-32.

14. Ziv I, Blackburn N, Rang M. Femoral intramedullary nailing in the growing child. J Trauma. 1984;24(5):432-4

15. Ulson HJR. Fraturas da extremidade distal do rádio e da ulna. In: Pardini Júnior AG. Traumatismos da mão. Rio de Janeiro: Medsi; 1985. p. 199-246.

16. Anastasopoulos J, Petratos D, Konstantoulakis C, Plakogiannis C, Matsinos G. Flexible intramedullary nailing in paediatric femoral shaft fractures. Injury. 2010;41(6):578-82.

17. Carey TP, Galpin RD. Flexible intramedullary nail fixation of pediatric femoral fractures. Clin Orthop Relat Res. 1996;(332):110-8. 